\title{
Covariant Effective Action for Quantum Particle with Coordinate-Dependent Mass
}

\author{
H. Kleinert* and A. Chervyakov ${ }^{\dagger}$ \\ Institut für Theoretische Physik, \\ Freie Universität Berlin, Arnimallee 14, 14195 Berlin, Germany
}

\begin{abstract}
Using a covariant background field method we calculate the one-loop quantum effective action for a particle with coordinate-dependent mass moving slowly through a one-dimensional configuration space. The procedure can easily be extended to any desired loop order.
\end{abstract}

\section{INTRODUCTION}

Just as the classical behavior of systems is completely determined by the extrema of the classical action, the quantum-mechanical properties can be found from the extrema of the so-called effective action. This can be calculated perturbatively in powers of the Planck constant $\hbar$ from the one-particle-irreducible (1PI) Feynman diagrams with no external quantum lines. The leading order is the classical action itself, whereas the one-loop term is a trace of a $\operatorname{logarithm}, \operatorname{Tr} \log M$, where $M$ is a functional matrix associated with small quantum fluctuations. The rules for calculating higher orders are well known.

Recently [1], the one-loop effective action was calculated for a particle in one dimension. Both the potential and the kinetic terms are modified by terms of order $\hbar$. The result is not applicable to a large variety of interesting physical systems, for instance compound nuclei, where the collective Hamiltonian, commonly derived from a microscopic description via time-dependent Hartree-Fock theory [2], contains coordinate-dependent mass parameters. This requires the extension of the effective action formalism to reparametrization-invariant quantum-mechanical systems in a curved spacetime similar to one-dimensional nonlinear $\sigma$-models.

A straightforward tool for computing the effective action of quantum-mechanical systems is provided by the background field method which in the context of quantum mechanics is described in the textbook [3]. In quantum field theory, this method provides a way to keep manifest the reparametrization invariance of $\sigma$-models, and is therefore the preferred method for explicit calculations of effective actions [1]. In this paper we use this method to calculate the effective action for a particle with coordinate-dependent mass. It is the simplest reparametrization-invariant quantum-mechanical $\sigma$-model with a one-dimensional parameter space. We generalize this model by including into the classical action a scalar potential. The model is UV-finite - all intermediate divergences will be seen to cancel against those coming from the invariant measure [5].

\section{BACKGROUND FIELD METHOD}

Consider a particle with coordinate-dependent mass $m(x)$ moving as in the one-dimensional potential $V(x)$. We shall study the euclidean version of the system where the paths $x(t)$ are continued to an imaginary times $\tau=-i t$ and the Lagrangian for $x(\tau)$ has the form

$$
\mathcal{L}(x, \dot{x})=\frac{1}{2} m(x) \dot{x}^{2}+V(x) .
$$

The dot stands for the derivative with respect to the imaginary time. The $x$-dependent mass may be written as $m g(x)$ where $g(x)$ plays the role of a one-dimensional dynamical metric. It is the trivial $1 \times 1$ Hessian matrix of the system. In $D$-dimensional configuration space, the kinetic term would read $m g_{\mu \nu}(x) x^{\mu} x^{\nu} / 2$ as in an $\mathrm{O}(D)$-symmetric nonlinear $\sigma$-model in one dimension.

Under an arbitrary single-valued coordinate transformation $x=x(y)$, the potential $V(x)$ is assumed to transform like a scalar whereas the metric $m(x)$ is a one-dimensional tensor of rank two:

$$
V(x)=V(x(y)) \equiv \widetilde{V}(y), \quad m(x)=\widetilde{m}(y) y^{\prime 2}(x) .
$$

\footnotetext{
*Email: kleinert@physik.fu-berlin.de URL: http://www.physik.fu-berlin.de/ ${ }^{\sim}$ kleinert

${ }^{\dagger}$ chervyak@physik.fu-berlin.de

On leave from LCTA, JINR, Dubna, Russia
} 
This coordinate transformation leaves the Lagrangian (2.1) and thus also the classical action

$$
\mathcal{A}[x]=\int_{-\infty}^{\infty} d \tau \mathcal{L}(x, \dot{x})
$$

invariant.

The quantum theory has to possess the same invariance. In Schrödinger theory, this is automatic. In the path integral formulation of the quantum system, this property is nontrivial, and only recently the authors have shown how to proceed to guarantee it. [5] Of course, this invariance must also be manifest in the effective action of the quantum theory. This will be achieved by combining the background field method 《⿶ with the techniques of Refs. [5]. In the background field method we split all paths into $x(\tau)=X(\tau)+\delta x(\tau)$, where $X(\tau)$ is the final extremal orbit and $\delta x$ describes the quantum fluctuations around it. At the one-loop level, the covariant effective action $\Gamma[X]$ becomes a sum of the classical Lagrangian $\mathcal{L}(X, \dot{X})$ and a correction term $\Delta \mathcal{L}$. It is defines by the path integral

$$
e^{-\Gamma[X] / \hbar}=\int \mathcal{D} \mu(\delta x) e^{-\frac{1}{\hbar}\left\{\mathcal{A}[X+\delta x]-\int d \tau \delta x \frac{\delta \Gamma[X]}{\delta X}\right\}},
$$

where the measure of functional integration $\mathcal{D} \mu(\delta x)$ is obtained from the initial invariant measure $\mathcal{D} \mu(x)=$ $Z^{-1} \prod_{\tau} d x(\tau) \sqrt{m(x)}$ and reads

$$
\mathcal{D} \mu(\delta x)=Z^{-1} \prod_{\tau} d \delta x(\tau) \sqrt{m(X)} e^{\frac{1}{2} \delta(0) \int d \tau \log \frac{m(X+\delta x)}{m(X)}},
$$

with $Z$ being some normalization factor. The generating functional (2.4) possesses the same symmetry under configuration space reparametrizations as the classical action (2.3).

We now calculate $\Gamma[X]$ in Eq. (2.4) perturbatively as a power series in $\hbar$

$$
\Gamma[X]=\mathcal{A}[X]+\hbar \Gamma_{1}[X]+\hbar^{2} \Gamma_{2}[X]+\ldots .
$$

The quantum corrections to the classical action (2.3) are obtained by expanding $\mathcal{A}[X+\delta x]$ and the measure (2.5) in powers of $\delta x$ :

$\mathcal{A}[X+\delta x]=\mathcal{A}[X]+\int d \tau \frac{D \mathcal{A}}{\delta X^{\mu}} \delta x^{\mu}+\frac{1}{2} \int d \tau \int d \tau^{\prime} \frac{D^{2} \mathcal{A}}{\delta X^{\mu} \delta X^{\nu}} \delta x^{\mu} \delta x^{\nu}+\frac{1}{6} \int d \tau \int d \tau^{\prime} \int d \tau^{\prime \prime} \frac{D^{3} \mathcal{A}}{\delta X^{\mu} \delta X^{\nu} \delta X^{\lambda}} \delta x^{\mu} \delta x^{\nu} \delta x^{\lambda}+\ldots$

The symbols $D / \delta X^{\mu}$ denote covariant functional derivatives. To first order, this is the ordinary derivative

$$
\frac{D \mathcal{A}[X]}{\delta X(\tau)}=\frac{\delta \mathcal{A}[X]}{\delta X(\tau)}=V^{\prime}(X)-\frac{1}{2} m^{\prime}(X) \dot{X}^{2}(\tau)-m(X) \ddot{X}(\tau) .
$$

This vanishes for the correct physical orbit $X(t)$. The second covariant derivative is

$$
\frac{D^{2} \mathcal{A}[X]}{\delta X(\tau) \delta X\left(\tau^{\prime}\right)}=\frac{\delta^{2} \mathcal{A}[X]}{\delta X(\tau) \delta X\left(\tau^{\prime}\right)}-\gamma(X) \frac{\delta \mathcal{A}[X]}{\delta X(\tau)} \equiv M\left(\tau, \tau^{\prime}\right)
$$

where $\gamma(X)=m^{\prime}(X) / 2 m(X)$ is the Christoffel symbol for the metric $m(X)$, and and the functional second derivative reads explicitly

$$
\frac{\delta^{2} \mathcal{A}[X]}{\delta X(\tau) \delta X\left(\tau^{\prime}\right)}=-\left[m(X) \frac{d^{2}}{d \tau^{2}}+m^{\prime}(X) \dot{X} \frac{d}{d \tau}+m^{\prime}(X) \ddot{X}+\frac{1}{2} m^{\prime \prime}(X) \dot{X}^{2}-V^{\prime \prime}(X)\right] \delta\left(\tau-\tau^{\prime}\right) .
$$

The validity of the expansion (2.7) follows from the fact that it is equivalent by a coordinates transformation to an ordinary functional expansion in Riemannian coordinates where the Christoffel symbol vanishes for the particular background coordinates.

The inverse of the functional matrix $M\left(\tau, \tau^{\prime}\right)$ in (2.9) supplies us with the propagator $G\left(\tau, \tau^{\prime}\right) \equiv M^{-1}\left(\tau, \tau^{\prime}\right)$. The higher derivatives define the interactions. The expansion terms $\Gamma_{n}[X]$ in (2.6) are found from all one-particleirreducible Feynman diagrams formed with the propagator $G\left(\tau, \tau^{\prime}\right)$ and the interaction vertices. The one-loop correction to the effective action is given by the simple harmonic path integral 


$$
e^{-\Gamma_{1}[X]}=\int \mathcal{D} \delta x \sqrt{m(X)} e^{-\mathcal{A}^{(2)}[X, \delta x]},
$$

with the quadratic part of the expansion (2.7):

$$
\mathcal{A}^{(2)}[X, \delta x]=\frac{1}{2} \int_{-\infty}^{\infty} d \tau d \tau^{\prime} \delta x(\tau) \frac{D^{2} \mathcal{A}[X]}{\delta X(\tau) \delta X\left(\tau^{\prime}\right)} \delta x\left(\tau^{\prime}\right) .
$$

The presence of $m(X)$ in the free part of the covariant kinetic term (2.12) and in the measure in Eq. (2.11) suggests exchanging the fluctuation $\delta x$ the new coordinates $\tilde{x}=h(X) \delta x$, where $h(X) \equiv \sqrt{m(X)}$ is the einbein $h(X)=\sqrt{m(X)}$ associated with the metric $m(X)$. Its covariant derivative is $D_{X} h(X)=\partial_{X} h(X)-\gamma(X) h(X) \equiv 0$. Then (2.12) becomes

$$
\mathcal{A}^{(2)}[X, \delta x]=\frac{1}{2} \int_{-\infty}^{\infty} d \tau d \tau^{\prime} \tilde{x}(\tau) \frac{D^{2} \mathcal{A}[X]}{\delta \tilde{X}(\tau) \delta \tilde{X}\left(\tau^{\prime}\right)} \tilde{x}\left(\tau^{\prime}\right)
$$

with

$$
\frac{D^{2} \mathcal{A}[X]}{\delta \tilde{X}(\tau) \delta \tilde{X}\left(\tau^{\prime}\right)}=e^{-1}(X) \frac{D^{2} \mathcal{A}[X]}{\delta X(\tau) \delta X\left(\tau^{\prime}\right)} e^{-1}(X)=\left[-\frac{d^{2}}{d \tau^{2}}+\Omega^{2}(X)\right] \delta\left(\tau-\tau^{\prime}\right)
$$

where

$$
\Omega^{2}(X)=e^{-1}(X) D^{2} V(X) e^{-1}(X)=e^{-1}(X) D V^{\prime}(X) e^{-1}(X)=\frac{1}{m(X)}\left[V^{\prime \prime}(X)-\gamma(X) V^{\prime}(X)\right] \equiv \tilde{M}\left(\tau, \tau^{\prime}\right) .
$$

Thus the fluctuations $\tilde{x}$ behave like those of a harmonic oscillator with the time-dependent frequency $\Omega^{2}(X)$, which is obviously a scalar under reparametrizations of configuration space. By construction, the fluctuation vanishes at the infinity $\tilde{x}(\tau)_{|\tau| \rightarrow \infty} \longrightarrow 0$. In the coordinates $\tilde{x}(\tau)$, the functional measure of integration in Eq. (2.11) takes the euclidean form

$$
\prod_{\tau} d \delta x(\tau) \sqrt{m(X)}=\prod_{\tau} d \tilde{x}(\tau) .
$$

This allows us to integrate the Gaussian path integral (2.11) trivially to obtain the one-loop quantum correction to the effective action

$$
\Gamma_{1}[X]=\frac{1}{2} \operatorname{Tr} \log \left[-d_{\tau}^{2}+\Omega^{2}(\tau)\right] .
$$

This expression is a nonlocal functional of the background field $X(\tau)$. It cannot be evaluated explicitly. For sufficiently slow movements of $X(\tau)$, however, we can resort to a gradient expansion which yields asymptotically a local expression for the effective action.

\section{DERIVATIVE EXPANSION}

The derivative expansion of the one-loop effective action (2.17) has the general form

$$
\Gamma_{1}[X]=\int_{-\infty}^{\infty} d \tau\left[V_{1}(X)+\frac{1}{2} Z_{1}(X) \dot{X}^{2}+\cdots\right]
$$

where $V_{1}(X)$ is the one-loop effective potential which can be found explictly by calculating the trace $\log$ in $(2.17)$ for a time-independent $\Omega^{2}$ :

$$
V_{1}(X)=\frac{1}{2} \operatorname{Tr} \log \left[-d_{\tau}^{2}+\Omega^{2}(\tau)\right]=\frac{1}{2} \int \frac{d k}{2 \pi} \log \left(k^{2}+\Omega^{2}\right)=\frac{1}{2} \Omega(X) .
$$

By construction, $V_{1}(X)$ and $Z_{1}(X)$ transform like a scalar and a tensor of rank 2 under reparametrizations of configuration space. In order to find $Z_{1}(X)$ we split the background field as $X(\tau)=X_{0}+\delta X(\tau)$, where $X_{0}$ is a constant 
field and $\delta X(\tau)$ is slowly varying, and expand (2.17) in powers of $\delta X$ up to $\delta X^{2}$. The general expression (3.2) shows that the second order expansion must have the form

$$
\Gamma_{1}\left[X_{0}+\delta X\right]-\Gamma_{1}\left[X_{0}\right]=\int_{-\infty}^{\infty} d \tau\left[D V_{1}\left(X_{0}\right) \delta X+\frac{1}{2} D^{2} V_{1}\left(X_{0}\right) \delta X^{2}+\frac{1}{2} Z_{1}\left(X_{0}\right) \delta \dot{X}^{2}\right]
$$

where

$$
\Gamma_{1}\left[X_{0}\right]=\frac{1}{2} \operatorname{Tr} \log \left[-d_{\tau}^{2}+\Omega^{2}\left(X_{0}\right)\right] .
$$

Such an expression is indeed obtained from the expansion of (2.17) which yields

$$
\Gamma_{1}\left[X_{0}+\delta X\right]-\Gamma_{1}\left[X_{0}\right]=\frac{1}{2} \operatorname{Tr} \log \left\{1+\frac{1}{\hat{k}^{2}+\Omega^{2}}\left[D \Omega^{2}\left(X_{0}\right) \delta X+\frac{1}{2} D^{2} \Omega^{2}\left(X_{0}\right)(\delta X)^{2}\right]\right\},
$$

where $\Omega^{2} \equiv \Omega^{2}\left(X_{0}\right)$ and we have introduced the wave number operator $\hat{k} \equiv-i d_{\tau}$. Expanding the logarithm in Eq. (3.5) up to $\delta X^{2}$, we obtain

$$
\begin{aligned}
\Gamma_{1}\left[X_{0}+\delta X\right]-\Gamma_{1}\left[X_{0}\right] & =\frac{1}{2} \operatorname{Tr}\left\{\frac{1}{\hat{k}^{2}+\Omega^{2}}\left[D \Omega^{2}\left(X_{0}\right) \delta X+\frac{1}{2} D^{2} \Omega^{2}\left(X_{0}\right)(\delta X)^{2}\right]\right\} \\
& -\frac{1}{4}\left(D \Omega^{2}\right)^{2}\left(X_{0}\right) \operatorname{Tr}\left[\frac{1}{\hat{k}^{2}+\Omega^{2}} \delta X \frac{1}{\hat{k}^{2}+\Omega^{2}} \delta X\right] .
\end{aligned}
$$

The term linear in $\delta X$ can immediately be calculated in the spectral representation of the propagator:

$$
\frac{1}{2} D \Omega^{2}\left(X_{0}\right) \int_{-\infty}^{\infty} d \tau \delta X(\tau) \int \frac{d k}{2 \pi} \frac{1}{k^{2}+\Omega^{2}}=\frac{1}{4 \Omega\left(X_{0}\right)} D \Omega^{2}\left(X_{0}\right) \int_{-\infty}^{\infty} d \tau \delta X(\tau) .
$$

We now recall the zeroth order term in Eq. (3.2) and rewrite this as

$$
\int_{-\infty}^{\infty} d \tau D V_{1}\left(X_{0}\right) \delta X(\tau)
$$

thus reproducing the the linear $\delta X(\tau)$-term in the expansion (3.3). In the calculation it is important that both $V_{1}(X)$ and $\Omega^{2}(X)$ are the scalars under reparametrizations, such that the first covariant derivatives are equal to the ordinary derivatives: $D V_{1}(X)=d V_{1}(X) / d X, D \Omega^{2}(X)=d \Omega^{2}(X) / d X$. The scalar nature of $\Omega^{2}(X)$ is obvious from the following covariant representation

$$
\Omega^{2}(X)=\frac{1}{\sqrt{m(X)}} \frac{d}{d X}\left[\sqrt{m(X)}\left(\frac{V^{\prime}(X)}{m(X)}\right)\right]
$$

which is the one-dimensional version of the Laplace-Beltrami expression $g^{-1 / 2} \partial_{\mu} g^{1 / 2} g^{\mu \nu} \partial_{\nu} V(X)$.

The quadratic terms in $\delta X$ in Eq. (3.6) read

$$
\frac{1}{4} D^{2} \Omega^{2}\left(X_{0}\right) \operatorname{Tr}\left[\frac{1}{\hat{k}^{2}+\Omega^{2}}(\delta X)^{2}\right]-\frac{1}{4}\left(D \Omega^{2}\right)^{2}\left(X_{0}\right) \operatorname{Tr}\left[\frac{1}{\hat{k}^{2}+\Omega^{2}} \delta X \frac{1}{\hat{k}^{2}+\Omega^{2}} \delta X\right] .
$$

The first functional trace is immediately calculated with the spectral representation of the propagator and yields

$$
\frac{1}{4} D^{2} \Omega^{2}\left(X_{0}\right) \int \frac{d k}{2 \pi} \frac{1}{k^{2}+\Omega^{2}}=\frac{1}{2} \frac{D^{2} \Omega^{2}\left(X_{0}\right)}{4 \Omega\left(X_{0}\right)} \int_{-\infty}^{\infty} d \tau(\delta X)^{2}(\tau) .
$$

The evaluation of the second term in (3.10) needs more work due to the $\tau$-dependence of $\delta X(\tau)$ between the differential operators. In order to use the spectral representation, we must move all operators $\hat{k}$ to the left of all $\delta X(\tau)$. Making use of the identity [6]

$$
\delta X \frac{1}{\hat{k}^{2}+\Omega^{2}}=\frac{1}{\hat{k}^{2}+\Omega^{2}} \delta X+\frac{1}{\left(\hat{k}^{2}+\Omega^{2}\right)^{2}}\left[\hat{k}^{2}, \delta X\right]+\frac{1}{\left(\hat{k}^{2}+\Omega^{2}\right)^{3}}\left[\hat{k}^{2},\left[\hat{k}^{2}, \delta X\right]\right]+\cdots
$$


and the obvious commutation relations

$$
\left[\hat{k}^{2}, \delta X\right]=\delta \ddot{X}-2 i \hat{k} \delta \dot{X}
$$

and

$$
\left[\hat{k}^{2},\left[\hat{k}^{2}, \delta X\right]\right]=\delta \ddot{\ddot{X}}-4 i \hat{k} \delta \dddot{X}-4 \hat{k}^{2} \delta \ddot{X}
$$

we reduce the second term in (3.10) to the following form

$$
-\frac{1}{4}\left(D \Omega^{2}\right)^{2}\left(X_{0}\right) \operatorname{Tr}\left[\frac{1}{\left(\hat{k}^{2}+\Omega^{2}\right)^{2}}(\delta X)^{2}\right]-\frac{1}{4}\left(D \Omega^{2}\right)^{2}\left(X_{0}\right) \operatorname{Tr}\left\{\left[\frac{1}{\left(\hat{k}^{2}+\Omega^{2}\right)^{3}}-\frac{4 \hat{k}^{2}}{\left(\hat{k}^{2}+\Omega^{2}\right)^{4}}\right] \delta X \delta \ddot{X}\right\}
$$

where the terms containing higher time derivatives of $\delta X(\tau)$ have to be omitted. Here the first term gives immediately

$$
-\frac{1}{4}\left(D \Omega^{2}\right)^{2}\left(X_{0}\right) \int \frac{d k}{2 \pi} \frac{1}{\left(k^{2}+\Omega^{2}\right)^{2}} \int d \tau(\delta X)^{2}(\tau)=-\frac{1}{2} \frac{\left(D \Omega^{2}\right)^{2}\left(X_{0}\right)}{8 \Omega^{3}\left(X_{0}\right)} \int_{-\infty}^{\infty} d \tau(\delta X)^{2}(\tau) .
$$

Combining this with (3.16), we find the quadratic term in $\delta X(\tau)$ :

$$
\frac{1}{2} D^{2} V_{1}\left(X_{0}\right) \int_{-\infty}^{\infty} d \tau \delta X^{2}(\tau)
$$

as follows directly from the definition $D^{2} V_{1}\left(X_{0}\right)=V_{1}^{\prime \prime}\left(X_{0}\right)-\gamma\left(X_{0}\right) V_{1}^{\prime}\left(X_{0}\right)=$ of Eq. 3.3$)$ and $V_{1}\left(X_{0}\right)=\Omega\left(X_{0}\right) / 2$.

The last term in (3.15) is

$$
-\frac{1}{4}\left(D \Omega^{2}\right)^{2}\left(X_{0}\right) \int \frac{d k}{2 \pi} \frac{\Omega^{2}-3 k^{2}}{\left(k^{2}+\Omega^{2}\right)^{4}} \int_{-\infty}^{\infty} d \tau \delta X(\tau) \delta \ddot{X}(\tau)=-\frac{\left(D \Omega^{2}\right)^{2}\left(X_{0}\right)}{64 \Omega^{5}\left(X_{0}\right)} \int d \tau \delta X(\tau) \delta \ddot{X}(\tau) .
$$

After a partial integration in $\tau$, this can be compared to last term in the gradient expansion (3.3). with the result

$$
Z_{1}\left(X_{0}\right)=\frac{\left(D \Omega^{2}\right)^{2}\left(X_{0}\right)}{32 \Omega^{5}\left(X_{0}\right)}
$$

We may now replace $X_{0}$ by $X(\tau)$ to obtain, finally, the one-loop correction to the kinetic term

$$
Z_{1}(X)=\frac{(D \Omega)^{2}(X)}{8 \Omega^{3}(X)} .
$$

Substituting this into with (3.2) into (3.1) and this into the expansion (2.6) we obtain effective action to order $\hbar$ :

$$
\Gamma^{\mathrm{eff}}[X]=\int_{-\infty}^{\infty} d \tau\left[\frac{1}{2} m^{\mathrm{eff}}(X) \dot{X}^{2}+V^{\mathrm{eff}}(X)\right],
$$

where the bare metric $m^{\text {eff }}$ and the potential $V^{\text {eff }}$ differ from the initial classical expressions by the above one-loop fluctuation corrections:

$$
\begin{aligned}
& m^{\mathrm{eff}}(X)=m(X)+\hbar \frac{(D \Omega)^{2}(X)}{8 \Omega^{3}(X)} \\
& V^{\mathrm{eff}}(X)=V(X)+\hbar \frac{1}{2} \Omega(X)
\end{aligned}
$$

The effective action has the same coordinate independence as the initial action. For an $x$-independent mass, the result reduces to that of Ref. [1].

A final word is necessary on the range of validity of the expansion. The derivation shows that the characteristic time scale is $1 / \Omega$. Within this time, the particle has to move only little, i,e., $\dot{X}(\tau) / X(\tau)$ has top be much smaller than $\Omega$. 


\section{CONCLUSION}

We have derived the one-loop approximation to the covariant effective action for a point particle with coordinatedependent mass moving slowly through a one-dimensional scalar potential. The extremization of this action yields an equation of motion whose solutions contain all quantum effects linear in $\hbar$.

[1] F. Cametti, G. Jona-Lasinio, C. Presilla and F. Toninelli, Comparison between quantum and classical dynamics in the effective action formalism, Proc. of the Int. School of Physics "Enrico Fermi", CXLIII Ed. by G. Casati, I. Guarneri, U. Smilansky, Amsterdam, IOS Press, 2000, pp. 431-448 quant-ph/9910065; B.R. Friedan and A. Plastino, Classical trajectories compatible with quantum mechanics, quant-ph/0006012 (2000).

[2] K. Goeke and P.-G. Reinhart, Ann. Phys. 112 (1978) 328.

[3] H. Kleinert, Path Integrals in Quantum Mechanics, Statistics and Polymer Physics, World Scientific Publishing Co., Singapore 1995, Second extended edition. For the original literature see G. 't Hooft, Nucl. Phys. B62 (1973) 444.

[4] L. Alvarez-Gaumé, D.Z. Freedman and S. Mukhi, Ann. of Phys. 134 (1981) 85;

see also:

J. Honerkamp, Nucl. Phys. B36 (1972) 130;

G. Ecker and J. Honerkamp, Nucl. Phys. B35 (1971) 481;

L. Tataru, Phys. Rev. D12 (1975) 3351;

G. A. Vilkoviski, Nucl. Phys. B234 (1984) 125;

E.S. Fradkin and A.A. Tseytlin, Nucl. Phys. B234 (1984) 509;

E. Braaten, T. L. Curtright and C. K. Zachos, Nucl. Phys. B260 (1985) 630;

P .S. Howe, G. Papadopoulos and K. S. Stelle, Nucl. Phys. B296 (1988) 26;

V. V. Belokurov and D. I. Kazakov, Particles \& Nuclei 23 (1992) 1322.

[5] H. Kleinert and A. Chervyakov, Phys. Lett. B464 (1999) 257;

hep-th/9906156;

H. Kleinert and A. Chervaykov, Phys. Lett. B477 (2000) 373; quant-ph/9912056.

[6] C. M. Fraser, Z. Phys. C28 (1985) 101; see also:

J. Iliopoulos, C. Itzykson, A. Martin, Rev. Mod. Phys. 47 (1975) 165;

K. Kikkawa, Prog. Theor. Phys. 56 (1976) 947;

H. Kleinert, Fortschr. Phys. 26 (1978) 565;

R. MacKenzie, F. Wilczek and A. Zee, Phys. Rev. Lett. 53 (1984) 2203;

I. J. R. Aitchison and C. M. Fraser, Phys. Lett. B146 (1984) 63. 